\title{
EXPERIMENTAL STUDY ON VOLTAGE DEPENDENT REACTIVE POWER CONTROL Q(V) BY SOLAR INVERTERS IN LOW-VOLTAGE NETWORKS
}

\section{Peter ESSLINGER}

Institute of Power Transmission Systems

Technische Universität München - Germany

peter.esslinger@mytum.de

\author{
Rolf WITZMANN \\ Institute of Power Transmission Systems \\ Technische Universität München - Germany \\ rolf.witzmann@tum.de
}

\begin{abstract}
This paper presents the results of an experimental study on voltage dependent reactive power control $Q(V)$ by solar inverters in low-voltage networks. The project's intention is a neutral investigation of the stability of a $V$ $Q$ control in low-voltage networks. The results of this study showed that a voltage dependent reactive power control $Q(V)$ by solar inverters in low-voltage networks will not cause any stability issues. Furthermore, this paper will comprise recommendations about the parameterization of the $V-Q$ control and about some important standardization issues.
\end{abstract}

\section{INTRODUCTION}

The proportion of renewable energies in power generation has increased significantly in recent years according to the political aims. In low-voltage networks, these are mostly distributed intermittent generation systems such as photovoltaic systems that are connected to the grid through inverters. Thus, the distribution networks have to meet hitherto unfamiliar technical challenges.

A temporary reversal of the power flow can cause overvoltage problems. Traditional voltage regulation strategies cannot solve this problem. Consequently, regenerative sources need to be turned off temporarily or on the long term the network infrastructure has to be extended by the network operator.

In some areas, the installed generation power is significantly higher than the consumption. Distributed generation (DG) causes altered power flow patterns. Thus, the power flow may even become bidirectional. The temporary reversal of the power flow can provoke voltage rises away from the substation, especially at remote feeder ends. If the voltage exceeds the tolerance of usually $10 \%$ [3] above nominal voltage, other devices and equipment might be damaged. In Germany a maximal voltage increase of $3 \%$ [4] caused by distributed power plants in low voltage networks is recommended. In case of reverse power flow, the maximum permitted voltage will be reached even below nominal power of the network branch. [5]

Therefore, the DG systems (e.g. photovoltaic (PV) inverters) have to take part in the voltage regulation. With the help of reactive power, which is generated by the solar inverters, power capability and voltage quality of the networks can be increased. Thus, network extension can be delayed or even avoided in many times.

In case of a closed-loop voltage control, the reactive power that the inverters absorb or inject depends directly on the voltage at the point of interconnection. A manual parameterization of the $V$ - $Q$-characteristics for each DG's inverter is not feasible because of the high penetration of DG in the distribution system (especially in Germany).

Universal $V$ - $Q$ characteristics that are valid for almost all network topologies are another solution. However, there are some concerns about stability of such a voltage dependent reactive power control $Q(V)$ by solar inverters in low-voltage networks.

That is why the German network operators EnBW Regional AG and E.ON Bayern AG plus the inverter manufacturers SMA Solar Technology AG, KACO new energy $\mathrm{GmbH}$ and Siemens AG joined in a cooperation project with the Institute of Power Transmission Systems of the Technische Universität München. The project's intention is a neutral investigation of the stability of a $V$ $Q$ control in low-voltage networks.

\section{EXPERIMENTAL SETUP}

The project's idea is to reproduce a branch of a lowvoltage network in an experimental setup. Three inverters feed into this branch.

\section{Reproduced network}

Based on a statistical analysis of several low voltage networks in southern Germany, typical and extreme networks were defined for rural, village and suburban areas. [1]

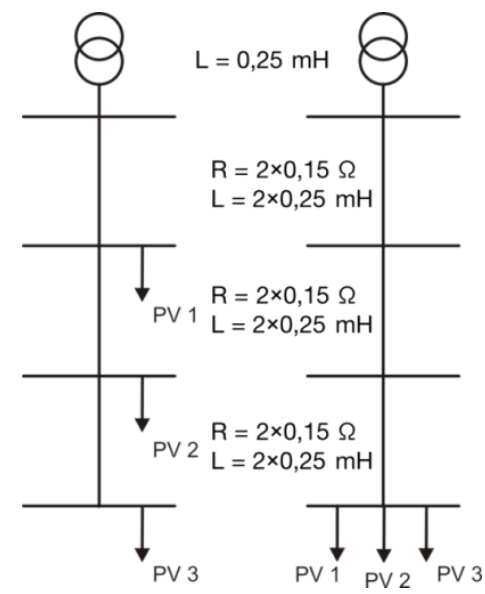

Figure 1: Parameters of the network branch

The typical networks are designed to represent the most common $50 \%$ of network topologies of each category. The extreme networks represent networks with e.g. long lines or high loaded transformers or a selection of network configurations which exist at a probability of only up to $5 \%$ but which are none the less considered as 
not negligible. This investigation focusses on rural networks because this category represents the most critical case concerning PV feed-in. Figure 1 shows the parameters $(R$ and $X)$ of the reproduced network branch which are based on [1] and [2]. This study comprises two arrangements of the inverters in the reproduced network branch: a longitudinal and a parallel one (cf. Figure 1).

\section{Inverters}

Three commercially available inverters with a rated power of $10 \mathrm{kVA}$ are used to be able to replicate situations as realistic as possible. Each of the three manufacturers which participated is this study provided one model. Specifically, these are the following types:

- SMA SUNNY TRIPOWER 10000TL

- KACO Powador 10.0 TL3

- Siemens SINVERT PVM 10

The inverters are able to realize a $Q(V)$ scheme, which is specified by a piecewise linear curve. The characteristic of this piecewise linear $Q(V)$ relation consists of at least three straight lines. These line segments can be configured with at least five points in the $Q-V$-plane (cf. example in Figure 2).

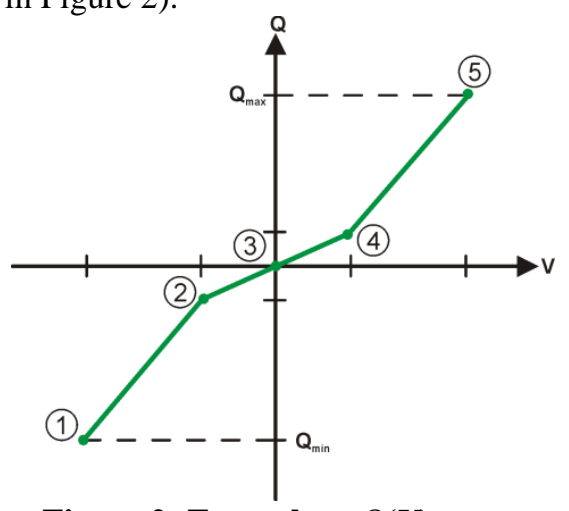

Figure 2: Exemplary $Q(V)$ curve

Additionally, a limitation of the maximum gradient of the reactive power is possible in order to avoid any instability by natural frequencies and measuring delays. Therefore, a PT1 element is integrated in the controller and can be optionally activated. In each case the time constant $T$ of the PT1 elements can be parameterized.

There is no default regarding the configuration and implementation of $Q(V)$ curves in inverters yet. Therefore, the inverters differ in the way how their control characteristics are specified in this study,. This does not affect the validity of the present study's results. As a side effect, the behaviour of $Q(V)$ control can be examined in a realistic situation with different implementations of the control system in the individual inverters.

Figure 3 shows the experimental setup. The inverter from KACO is on the left side and electrically at the end of the network branch. The inverter from SMA is in the centre and the inverter from Siemens is on the right side and electrically at the beginning of the network branch. The position the inverters was random and can be changed independently of the mechanical test setup. The lower part of Figure 3 shows the DC power supplies that drive the inverters. On top of the image follow measuring units and terminal blocks.

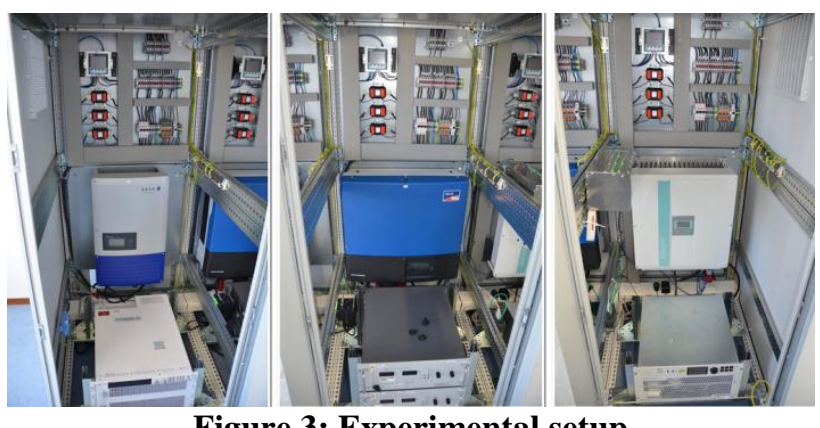

Figure 3: Experimental setup

The reproduced low-voltage network is on top of these installations and shown in Figure 4. In the extreme case, the left and the right part are connected in series and in the typical case, the right-hand part bridged.

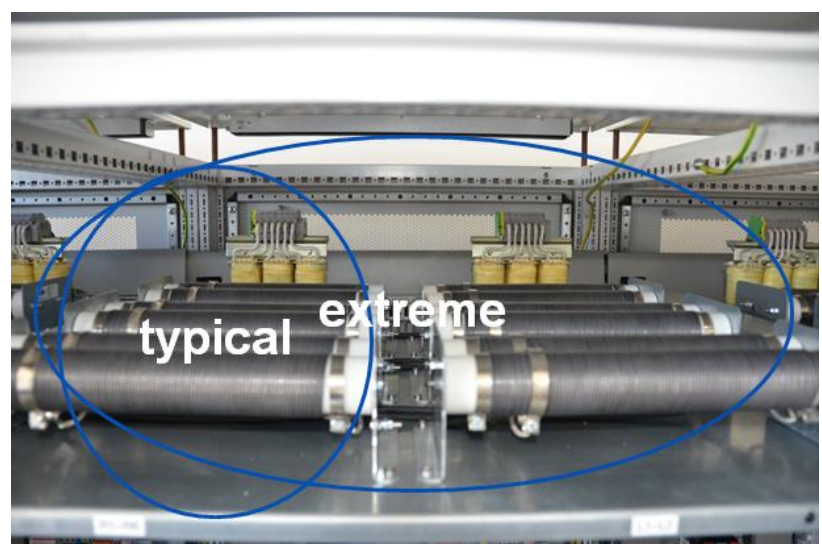

Figure 4: Reproduced low-voltage network

\section{REALIZATION OF THE STUDY}

This study was carried out with three different profiles:

\section{Jump}

It is necessary to know the inverters' step responses in order to investigate their control performance. Therefore, a first test series in which the inverters were supplied individually with jumps of the active power was conducted. Figure 5 shows the used feed-in profile.

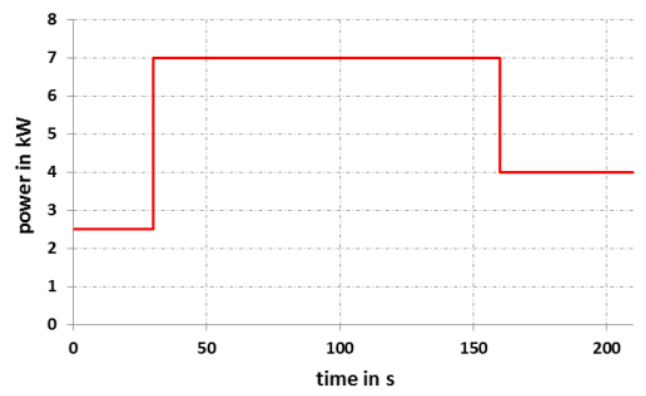

Figure 5: Profile Jump

The profile does not start at $0 \mathrm{~kW}$, because the inverters 
need some time for their initialisation procedure. Thus, all DC power supplies were set at a power output of $2.5 \mathrm{~kW}$ in order to initialise the inverters.

\section{Ramp}

The control behaviour of all three inverters in the entire system was first tested with a ramp-like profile of the active power feed-in. In this case, the control behaviour can be easily understood and validated. In this way the control behaviour on nice sunny days can be investigated. If there are no signs of unstable behaviour with the used ramp profiles, no unstable behaviour is to be expected even on sunny days, where far less power gradients occur. Figure 6 shows the used ramp profile.

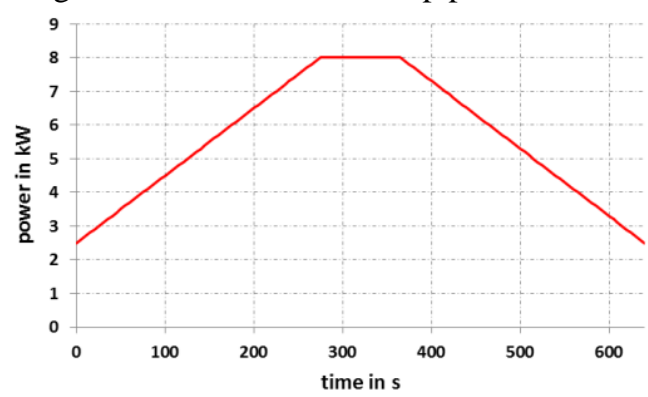

Figure 6: Ramp profile

\section{Volatile day}

Real measured PV feed-in profiles from a PV power plant were used to investigate the behaviour of the $Q(V)$ control on a volatile day (i.e. a sunny day with many passing clouds). In this case there are comparatively very large changes in the power feed-in. If the control scheme is in this situation is stable, no further instabilities are expected in real use. The measured values of the PV system are recorded with a time step of $1 \mathrm{~s}$. In this study, the DC power supplies feed the inverters with a time step of $5 \mathrm{~s}$. Figure 7 shows the used feed-in profile to. It clearly shows that with a time step of $5 \mathrm{~s}$ no substantial information is lost.

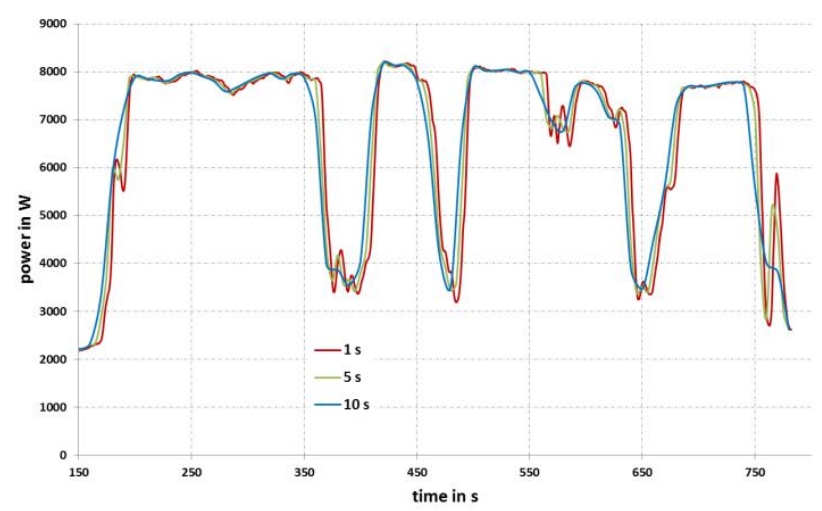

Figure 7: Profile volatile day

\section{Further experiments}

Moreover, different specific experiments were conducted in order to proof the stability of a $Q(V)$ control in further cases:

- Longitudinal and parallel arrangement of the inverters in the reproduced branch (cf. Figure 1)

- Experiment with passing clouds, i.e. there is a time shift in the inverters' profiles

- Experiments with a one-phase load

\section{RECOMMENDATIONS}

\section{Parameterization}

This project investigated the stability behaviour of the $Q(V)$ control. The aim was to reproduce particular critical cases and to investigate them. There was no evidence for any instability. Thus, regarding the required stability of the overall system, there are no strict restrictions on the choice of the parameters of the $Q(V)$ characteristics.

Nevertheless, resulting from practical considerations, there are some suggestions for setting parameters of a $Q(V)$ curve, which will be presented briefly below:

Basically, the setting of $Q(V)$ characteristics of $\mathrm{PV}$ inverters in low voltage networks depends on how the network operator operates the voltage band in the medium- and low-voltage networks. The control's parameterization in the medium and low voltage networks have to be coordinated according to the particular network environment and the philosophy of the network operator.

For example, a voltage reduction could be achieved by under excited (leading) operation of the DG's inverter beginning from a voltage of 1.05 p.u. Taking into account a measurement tolerance of $1 \%$, the maximum reactive power should be achieved at 1.09 p.u. in order to ensure the maximum voltage reduction effect before the response of the surge protector. The curve between these two points is linearly interpolated. As default settings for the overexcited (lagging) operating range of the inverters no reactive power at all (cf. Figure 8), a symmetric (cf. Figure 9) or an asymmetric (cf. Figure 10) characteristic would be possible.

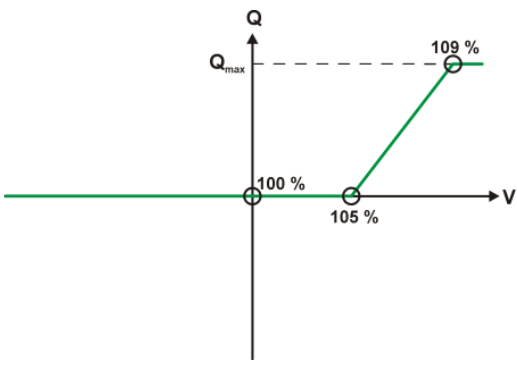

Figure 8: $Q(V)$ characteristic, only voltage reducing

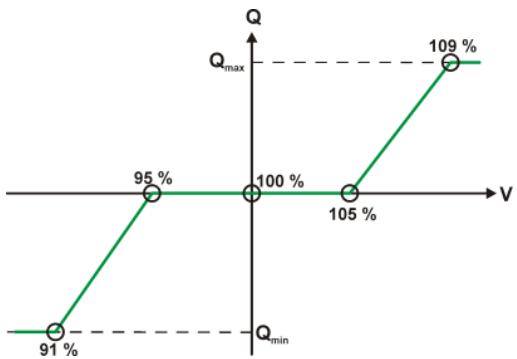

Figure 9: Symmetric $Q(V)$ characteristic 


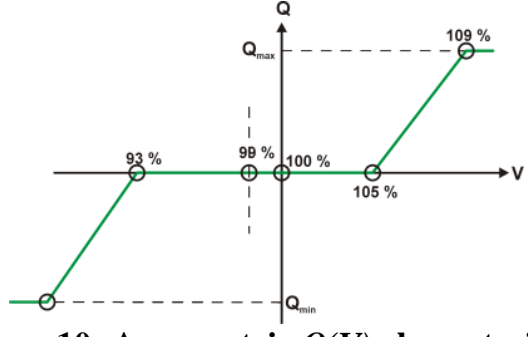

Figure 10: Asymmetric $Q(V)$ characteristic

Because of the limited rise time of the reactive power, there are brief increases of the voltage after voltage jumps, which are reduced when the inverter start to absorb reactive power. This is systemic, but not a sign of instability of the $Q(V)$ control. It is therefore recommended not to choose too large time constants for the PT1 element in order to enable the compensation of typical voltage gradients, as they occur with PV power plants. A time constant of the PT1 element of $5 \mathrm{~s}$ already calms the course of the reactive power considerably. Too small time constants should also not be selected, as they increase the sensitivity of the system unnecessarily.

Generally, it is recommended for DG (PV, CHP, etc.) in low-voltage networks that the $Q(V)$ control should be a little (ca. one to five times) faster than the maximum expected power change $\mathrm{d} P / \mathrm{d} t$ of the DG. However, a $Q(V)$ control which is too fast (more than ten times) is also not a good parameterization. The time constant of the PT1 element should also not be too large in order to achieve a decoupling of the $Q(V)$ control in medium voltage.

\section{Standardization}

The experiments with the three different inverters showed some different possibilities for an implementation of the control system behaviour, which, although they meet the requirements of the German guideline VDE-AR-N 4105, should be standardized.

Parameterization and verification of the $Q(V)$ control There are different ways to parameterise the $Q(V)$ control implemented in the PV inverters. This can lead to difficulties in the correct implementation of control specifications. To avoid possible problems and confusion associated with parameterization, it is necessary to develop a standard for specifying the $Q(V)$ (e.g. 5 pairs of values or line segments, etc.). When preparing the unit certificates (VDE V 0124-100) there should be a proof of if the scheme is in accordance with the characteristic setting.

\section{Relevant voltage for the $Q(V)$ control}

Up to now, it is not explicitly standardised which voltage is relevant for the $Q(V)$ control of three-phase inverters. Currently, solutions implemented in the PV inverters' control system depend on the inverters' manufacturers. There are two main approaches: the mean of the phase-toearth voltages and the maximum of the phase-to-earth voltages. Other options would include the positive sequence voltage or the medium or maximum phase voltages. An advantage of the use of the voltage mean value might be a less sensitive control behaviour. A scheme based on the positive sequence voltage appears rather inappropriate because it is more complex to implement and needs more computing time. Furthermore, it is difficult to determine if there are any benefits. The variations should be further clarified in order to avoid deviant control behaviour which can be observed in some areas, even though, this is not relevant for the functioning and stability of the overall system.

$Q(V)$ control of one-phase inverters and one-phase $Q(V)$ control of three-phase inverters

The experiments were carried out only with three-phase inverters. Thus, it was ensured that the reactive power control is symmetric (positive sequence) in accordance with the requirements of the German guideline VDE-AR$\mathrm{N}$ 4105. Therefore, already existing asymmetrical current and voltage conditions in the low-voltage network are not adversely affected. It would also be possible that inverter systems control the voltage on each phase or specifically counteract voltage asymmetry (negative sequence voltage). These methods are promising, but also cause unbalanced currents and reactive power flows in the lowvoltage network. The advantages and disadvantages thereof and the impact on the protection systems (no overcurrent protection on neutral) should be investigated in further studies. Accordingly, the $Q(V)$ control of onephase inverters, which can also control the voltage according to one phase only, should be examined closer.

\section{CONCLUSION}

The project showed that a voltage dependent reactive power control $\mathrm{Q}(\mathrm{V})$ by solar inverters in low-voltage networks will not cause any stability issues. Furthermore, this paper comprises recommendations about the parameterization of the V-Q control and about some important standardization issues.

\section{REFERENCES}

[1] G.Kerber, R. Witzmann, Statistical Distribution Grid Analysis and Reference Network Generation, ew, 2007

[2] G.Kerber, R. Witzmann, Voltage Limitation by Autonomous Reactive Power Control of Grid Connected Photovoltaic Inverters, CPE 2009, 6th International Conference-Workshop Compatibility and Power Electronics, Badajoz, Spain, May 20 22, 2009

[3] Voltage characteristics of electricity supplied by public distribution systems, EN 50160, 2007

[4] Generators connected to the low-voltage distribution networkTechnical requirements for the connection to and parallel operation with low-voltage distribution networks, VDE-AR-N 4105, 2011

[5] G. Kerber, Capacity of Low Voltage Distribution Networks Due to Power generation of Small Photovoltaic Power Plants, Ph.D. dissertation, Institute of power transmission systems, Technische Universität München, 2011

\section{FURTHER INFORMATION}

A detailed report of this study in German language is available online at https://mediatum.ub.tum.de/ download/1114994/1114994.pdf 